\title{
Numerical heat transfer model in skin burn depth simulations
}

\author{
By M.Bajorek, M.Kaczmarek*
}

${ }^{*}$ Department of Biomedical Engineering Gdansk University of Technology, Gdansk, Poland

\section{Abstract}

The aim of this paper is to discuss properties of the finite difference method in numerical simulations of heat transfer in skin. The analysis is done for the proposed model implemented in Borland $\mathrm{C}_{++}$Builder, for static and transients conditions and external excitation. The results of simulation are compared to the simulation results in I-DEAS® Finite Element Modeling (FEM) and to thermograms recorded in animal experiments. Numerical model is implemented for one and three-dimensional heat transfer model.

\section{Introduction}

The aim of this work is to optimize finite difference method to heat transfer simulation in skin. Presently performing correct evaluation of skin burn depth in order to make the appropriate choice of treatment is one of the most important unsolved problems in skin burn diagnostics [1]. In the absence of any objective procedure allowing quantitative evaluation of a skin burn depth, current research interests have concentrated on the search for new, noninvasive and quantitative diagnostic methods. One of this methods is thermal tomography. numerical simulation of surface temperature distributions for different skin model thermal parameters combined with ADT experiments[2]. The main disadvantages of currently used heat transfer simulation programs are: very long time of simulations which eliminates this solutions from real time diagnostic and general heat transfer equations which not include all thermal processes occurring in human skin. To solve mentioned problems a special software in Borland $\mathrm{C}_{++}$ Builder environment was implemented with modified simulation algorithm based on finite difference method and the extended heat transfer equation for biological tissue. Changes connected with blood perfusion were included (according to Pennes[3] and modified by Buettner[4]). The proposed model was examined in terms of computational mesh complexity on time and accuracy of simulations.

The geometry of the used skin model is presented in Fig1. Thermal parameters of the skin tissue accepted in numerical simulations are in details described in [2]. Healthy skin model and superficial burn wound is consist of five layers, while the model of the deep burn wound is composed of only two layers, because of much greater damage of the wound tissue and blurring of its layer structure. For simplification structure of the layers of the models is assumed to be homogeneous and isotropic, without veins, arteries and hair follicles.

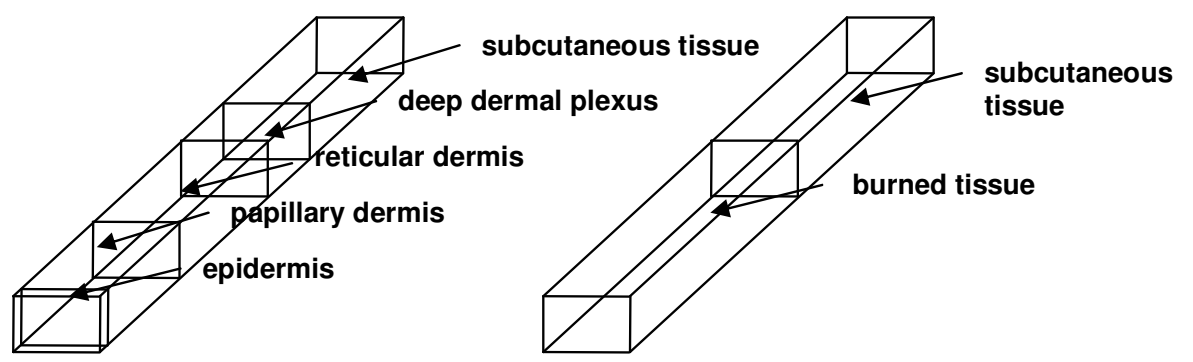

Fig. 1. Geometry of thermal skin models: (a)for healthy skin and for a superficial burn wound, (b) for a deep burn wound

\section{Implementation}

In the first step the discretization of space and time is made, such that there is an integer number of nodes in the space (elementary volumes of material) and an integer number of times at which we calculate temperature distribution. The basic modelling idea is to compute temperature for all the nodes, taking into account thermal properties of the material and the thermal exchanges between the nodes themselves and the environment. The numerical equation for internal nodes obtained through application finite difference method to Fourier's heat transfer equation. The first time derivative was approximated by the forward difference equation, the second space derivative was approximated by using the above central difference formula:

$$
T_{i}^{n+i}=(\Delta F o) T_{i-1}^{n}+[1-2(\Delta F o)] T_{i}^{n}+(\Delta F o) T_{i+1}^{n} .
$$
In order to find particular solutions initial and boundary conditions for solving transient problems are required. Boundary conditions define how solution behaves along external surfaces of the body. In the 
considered case where the reaction of healthy and burned pigskin to forced external cold excitation is measured, external nodes are completed with boundary Robin`s condition:

$$
-\left.\lambda \frac{\partial T}{\partial x}\right|_{x=0}=\alpha_{1}\left(\left.T\right|_{x=0}-T_{c z, 1}\right) \text {. }
$$

Temperature in external nodes is computed after using finite difference formulas.

$$
T_{1}^{n+i}=2(\Delta F o)\left[T_{2}^{n}+\left(\Delta B_{i}\right) T_{c z, 1}^{n}\right]+\left[1-2(\Delta F o)+2\left(\Delta B_{i}\right)(\Delta F o)\right] T_{1}^{n} .
$$

\section{Results}

For testing of the proposed numerical heat transfer model the following assumptions were made: constant core temperature for deeper tissue $T_{c}=38.5 \stackrel{\circ}{\circ}$, external cooling is simulated as air flow in temperature $T_{\text {air flow }}=0^{\circ} \mathrm{C}$ lasting from $10 \mathrm{~s}$ to $30 \mathrm{~s}$, heat exchange between skin surface and the atmosphere after the external cooling realized as free convection with parameter $T_{\text {air }}=23^{\circ} \mathrm{C}$. Calculations are performed for the period of external cooling and the natural heating phase $(30 \mathrm{~s})$, after external stimulus is switched off. The implemented model was examined for researching dependence of time of numerical simulations from size of simulated surface and discretization level. Parameters and part of simulations results are gathered in Table 1. Table 2 presents influence of computational mesh complexity on accuracy of simulated temperature.

Table 1. Dependence of simulation time from complexity of generated mesh

\begin{tabular}{|l|l|l|l|l|l|}
\hline Size & $\begin{array}{l}\text { Number of } \\
\text { surface points }\end{array}$ & $\begin{array}{l}\text { Steps of } \\
\text { discretization }\end{array}$ & $\begin{array}{l}\text { Simulated } \\
\text { time [s] }\end{array}$ & $\begin{array}{l}\text { Main loop repetition } \\
\text { number }\end{array}$ & $\begin{array}{l}\text { Numerical } \\
\text { simulation time[s] }\end{array}$ \\
\hline $20 \times 20$ & 400 & 23 & 10 & 92000000 & 158 \\
\hline $40 \times 40$ & 1600 & 23 & 10 & 368000000 & 200 \\
\hline $80 \times 40$ & 3200 & 23 & 10 & 736000000 & 293 \\
\hline $120 \times 80$ & 9600 & 23 & 10 & 2208000000 & 551 \\
\hline $160 \times 120$ & 19200 & 23 & 10 & 4416000000 & 938 \\
\hline $320 \times 240$ & 76800 & 23 & 10 & 17664000000 & 3360 \\
\hline
\end{tabular}

Table 2. Dependence of temperature accuracy from complexity of generated mesh for healthy skin model.

\begin{tabular}{|c|c|c|}
\hline Steps of discretization & Average error $\left[{ }^{\circ} \mathbf{C}\right]$ & Maximum error $\left[{ }^{\circ} \mathbf{C}\right]$ \\
\hline $36(3 ; 5 ; 10 ; 10 ; 8)$ & 0,007 & 0,017 \\
\hline $36(3 ; 5 ; 10 ; 8 ; 10)$ & 0,015 & 0,034 \\
\hline $36(3 ; 5 ; 8 ; 10 ; 10)$ & 0,22 & 0,40 \\
\hline $33(3 ; 5 ; 5 ; 10 ; 10)$ & 0,69 & 1,21 \\
\hline $28(3 ; 5 ; 5 ; 5 ; 10)$ & 0,73 & 1,31 \\
\hline $23(3 ; 5 ; 5 ; 5 ; 5)$ & 0,76 & 1,39 \\
\hline
\end{tabular}

\section{Discussion and conclusions}

The first simulations show that using optimized finite difference method to heat transfer simulations allows to get reliable results of heat process in skin tissue. This method can give objective information for skin burn diagnostics. Specially promising results are for high computational mesh complexity but high accuracy computations require long simulation time. Currently all works are concentrated on further optimization of the algorithm.

\section{REFERENCES}

[1] P. G. Shakespeare, "Looking at burn wounds: The A. B. Wallace memorial lecture," Burns 1992, vol. 18, pp. 287-295,1991.

[2] Ruminski J., Kaczmarek M, Renkielska A, Nowakowski A., Thermal Parametric Imaging In the Evaluation of Skin Burn Depth, IEEE Transactions of Biomedical Engineering, Vol.54, No. 2, p.

[3] Pennes H.H. analysis of tissue and arterial blood temperatures in resting human forearm. J. Appl. [Physiol. 1, $93-122,1947$

[4] Buettner K., Effects of extreme heat and cold human skin. I. Analysis of temperature changes caused by different kind of heat application, Journal of Applied Physiology, Vol. 3, No 12, p. 691 713,1951 . 